Pacific Journal of Mathematics

EXTENDED PRIME SPOTS AND QUADRATIC FORMS 


\section{EXTENDED PRIME SPOTS AND QUADRATIC FORMS}

\section{RON BROWN}

Some of the local theory of extended prime spots on fields is developed here, with two applications in mind. In the first, two analogues to the Hasse-Minkowski theorem on equivalence of quadratic forms over global fields are developed, based on the notion of an ultracompletion of a field at an extended prime spot. They deal, respectively, with equivalence of quadratic forms over a simple transcendental extension of a global field, and with the reduced Witt ring of a general field. Examples illustrate problems involving the further extension of the global theory of quadratic forms. In the second application Harrison and Warner's ultracompletions of a field at a finite or infinite prime are shown to be essentially ultracompletions at associated extended prime spots.

Extended prime spots were first introduced to provide a setting for a generalization of the weak approximation theorem for independent absolute values. In $\S 1$, we recall from [3] the definitions of extended absolute values and extended prime spots and we define and study ultracompletions of fields at extended absolute values. An alternative approach to ultracompletions of fields at Harrison primes [7] is sketched in $\S 2$. The analogues to the Hasse-Minkowski theorem are discussed in $\S 3$; this section owes a great debt to work of Milnor [14] and Pfister [15]. Finally, in an appendix (\$4) we sketch a theory of Henselizations of extended absolute values. The last three sections of this paper are essentially independent of each other.

Throughout this paper $F$ will denote a field. $F^{\times}$denotes its multiplicative group of nonzero elements. $Z, Q, R$, and $C$ denote the sets of integers, rational numbers, real numbers and complex numbers, respectively. $A \backslash B$ denotes the set of elements of the set $A$ which are not in the set $B$.

1. Ultracompletions. We recall some concepts from [3, especially §5]. An extended absolute value on $F$ is a map $\varphi: F \rightarrow R \cup\{\infty\}$ with $\varphi(a+b) \leqq \varphi(a)+\varphi(b), \varphi(a b)=\varphi(a) \varphi(b)$ (when defined), $\varphi(a) \geqq 0$, $\varphi(1)=1$ and $\varphi(0)=0$ for all $a, b \in F$. (We do not define $0 \cdot \infty$ or $\infty$.0.) The extended absolute values on $F$ are precisely those maps obtained by composing a place on $F$ with an absolute value on the residue class field of the place. (We intend that the composite function map to $\infty$ those elements of $F$ which the place maps to $\infty$.) For, if $\varphi$ is an extended absolute value on $F$, then $\varphi^{-1}(R)$ is a valua- 
tion ring and $\varphi$ induces an absolute value on its residue class field. The following examples will be used below.

EXAMPLE 1.1. (A) An absolute value is a fortiori an extended absolute value.

(B) The valuation rings of $F$ are naturally bijective with the extended absolute values of $F$ mapping into $\{0,1, \infty\}$ (assign to each such extended absolute value $\phi$ the valuation ring $\varphi^{-1}(R)$ ).

(C) A place from $F$ into $C$ has canonically associated with it an extended absolute value (compose the place with the ordinary absolute value on $C$ ).

(D) An ordering of $F$ gives rise canonically to a place from $F$ into $R$ [2] and hence to an extended absolute value (cf. (C) above). (By an "ordering of $F$ " we mean the set of nonnegative elements in a total order on $F$ making $F$ an ordered field.) Similarly, a generalized power series field [8, p. 314] with residue class field $R$ or $C$ has canonically associated with it a complex place, and hence an extended absolute value. (Indeed, one might argue that the extended absolute value is an essential part of what is meant by a "generalized power series field with residue class field $R$ or $C$ ".)

The prime values [3, Definition 1.1] of $F$ are precisely the extended absolute values associated with either a complex place or a minimal valuation ring (cf. (C) and (B) above, respectively). The normalized extended absolute values [3, Definition 5.3] are simply those associated with a complex place or a valuation ring.

For the remainder or this section, $\varphi$ denotes an extended absolute value on $F$. We set

$$
D_{\varphi}=\{a \in F: \varphi(a)<1\} .
$$

DeFinition 1.2. A $\varphi$-topology on $F^{\times}$is a (not necessarily Hausdorff) group topology on $F^{\times}$admitting a neighborhood basis at 1 of the form $\left\{1+a D_{\varphi}: a \in A\right\}$ where $A$ is a nonempty subset of $F^{\times}$. (For group topologies see, for example, [10].)

Definition 1.3. $(F, \varphi)$ is ultracomplete (or, $F$ is ultracomplete at $\varphi$ ) if and only if $F^{\times}$is topologically complete in every $\varphi$-topology.

The word "ultracomplete" was first used, I think, by Fleischer in a related context [5].

Definition 1.4. A subfield $E$ of $F$ is ultradense in $(F, \varphi)$ if and only if $E^{\times}$is dense in $F^{\times}$with respect to some $\varphi$-topology.

Definition 1.5. $(F, \varphi)$ is an ultracompletion of a subfield $E$ of $F$ if and only if $(F, \varphi)$ is ultracomplete and $E$ is ultradense in $(F, \varphi)$. 
In which case, we also say both that $(F, \varphi)$ is an "ultracompletion of $(E, \varphi \mid E)$ " and that $(F, \varphi)$ is an "ultracompletion of $E$ at $\varphi \mid E$ ". (Here $\varphi \mid E$ denotes the restriction of $\varphi$ to $E$.)

The existence, uniqueness, and structure of ultracompletions is discussed in Remark 1.15.

Recall that $\varphi$ is equivalent to an extended absolute value $\psi$ when $D_{\varphi}=D_{\psi}$. If $\varphi$ and $\psi$ are equivalent, then every $\varphi$-topology on $F^{\times}$ is a $\psi$-topology, and vice versa. Hence the above concepts could have been defined for equivalence classes of extended absolute values, and, in particular, for extended prime spots. (An extended prime spot is an equivalence class of maximal extended absolute values, where we preorder the extended absolute values on $F$ by the rule $\varphi \leqq \psi$ if and only if $D_{\varphi} \subseteq D_{\psi}$. The prime values are precisely the normalized elements of the extended prime spots [3, §5].)

Example 1.6. Suppose $F$ is a global field. Then $\varphi$ is equivalent to an absolute value. The concepts of ultracompletion, ultracompleteness and ultradensity for $(F, \varphi)$ reduce to those of completion, completeness and density with respect to the metric topology of any such absolute value.

Recall that $\varphi^{-1}(R)$ is a valuation ring; $\varphi^{-1}(0)$ is its maximal ideal. Let $k_{\varphi}$ and $\Gamma_{\varphi}$ denote the residue class field and value group of $\varphi^{-1}(R)$, respectively. Let $\bar{\varphi}$ denote the absolute value induced by $\varphi$ on $k_{\varphi}$.

THEOREM 1.7. The following statements are equivalent:

(1) $(F, \varphi)$ is ultracomplete;

(2) $F$ is ultradense in no proper extension of $(F, \varphi)$;

(3) $\varphi^{-1}(R)$ is maximal and $\left(k_{\varphi}, \bar{\varphi}\right)$ is ultracomplete.

$\varphi^{-1}(R)$ is called maximal if the valuation it induces on $F$ makes $F$ a maximal field [16]. (Equivalently: $F$ with the discrete topology is a linearly compact $\varphi^{-1}(R)$-module $[16$, Chapter $\mathrm{D}$, Theorem 4 (Zelinsky)]. Also equivalently: $F^{\times}$is complete in every group topology with a neighborhood base at 1 of the form $\left\{1+b \varphi^{-1}(0): b \in B\right\}$ where $B \subseteq F^{\times}$[4, Example 2.2].) An extension of $(F, \varphi)$ is a pair $(K, \psi)$ consisting of a field $K$ containing $F$ and an extended absolute value $\psi$ on $K$ restricting to $\varphi$ on $F$. If $(K, \psi)$ is an extension of $(F, \varphi)$, then we may regard $\left(k_{\psi}, \bar{\psi}\right)$ as an extension of $\left(k_{\varphi}, \bar{\varphi}\right)$ and $\Gamma_{\varphi}$ as a subgroup of $\Gamma_{\psi}$.

THEOREM 1.8. $F$ is ultradense in an extension $(K, \psi)$ of $(F, \varphi)$ if and only if $k_{\varphi}$ is ultradense in $\left(k_{\psi}, \bar{\psi}\right)$ and $\Gamma_{\varphi}=\Gamma_{\psi}$. 
We will prove Theorems 1.7 and 1.8 in the course of the following remarks. We call $\varphi$ Archimedean if $\varphi(1+1)>1$, and nonarchimedean otherwise. ( $\varphi$ is nonarchimedean if and only if $\varphi(a+b) \leqq$ $\max \{\varphi(a), \varphi(b)\}$ for all $a, b \in F$.) One can check that $\varphi$ is Archimedean (nonarchimedean, normalized, a prime value) if and only if $\bar{\phi}$ is Archimedean (respectively, nonarchimedean, normalized, a prime value).

REMARK 1.9. We examine the ultracompletions of $\left(k_{\varphi}, \bar{\varphi}\right)$. Our remarks would hold for any field with an absolute value, of course.

First suppose $\varphi$ is Archimedean (or normalized!). Then the only $\bar{\varphi}$-topology on $k_{\varphi}^{\times}$is the metric topology of $\bar{\varphi}$. The ultracompletions of $\left(k_{\varphi}, \bar{\varphi}\right)$ are simply the topological completions of $k_{\varphi}$ in the metric topology. Ultradensity and ultracompleteness similarly reduce to density and completeness. Recall that every field complete at an Archimedean absolute value is isomorphic to $R$ or $C$.

Now suppose $\varphi$ is nonarchimedean, so $\bar{\varphi}$ is a valuation. Then the ultracompletions of $\left(k_{\varphi}, \bar{\varphi}\right)$ are simply the maximal immediate extensions of $k_{\varphi}$ (as a field with the valuation $\bar{\varphi}$ ). Ultradensity and ultracompleteness reduce to immediacy and maximality, respectively. (See [4, Example 2.2].)

Note that $\left(k_{\varphi}, \bar{\varphi}\right)$ is ultracomplete if $\varphi$ is normalized and nonarchimedean (since then $D_{\bar{\varphi}}=\{0\}$ ).

Lemma 1.10. Suppose $B \cong F^{\times}$(possibly $B$ is empty). There is a unique coarsest $\varphi$-topology separating 1 from $1+b$ for all $b \in B$. The collection of sets of the form

$$
1+b n^{-1} D_{\varphi}(b \in B \cup\{1\}, n \in Z \quad \text { with } \quad \varphi(n \cdot 1) \geqq 1)
$$

is a neighborhood base at 1 for this topology.

Proof. Note $1+n^{-1} D_{\varphi} \leqq 1+b n^{-1} D_{\varphi}$ if $\varphi(b) \geqq 1$. Suppose $b \in$ $B \cup\{1\}, n \in Z$ and $\varphi(b)<1 \leqq \varphi(n \cdot 1)$. If $\varphi$ is Archimedean, pick $r \in Z$ with $3 n / r \in D_{\varphi}$. Otherwise, set $r=n$. Then $\varphi(r) \geqq 1$ and $\left(1+b r^{-1} D_{\varphi}\right)^{2}$ and $\left(1+b r^{-1} D_{\varphi}\right)^{-1}$ are contained in $1+b n^{-1} D_{\varphi}$. It follows that the collection of sets (1) forms a neighborhood base a 1 for a $\varphi$-topology on $F^{\times}$. Clearly this topology separates 1 from $1+b$ for all $b \in B$. We will show it is contained in every other such $\varphi$-topology.

Let $\mathscr{T}$ now be any $\varphi$-topology separating 1 from $1+b$ for all $b \in B$. Let $b \in B$ and $n \in Z$ have $\varphi(b) \leqq 1 \leqq \varphi(n \cdot 1)$. We must show $1+b n^{-1} D_{\varphi}$ contains an open (with respect to $\mathscr{T}$ ) neighborhood of 1 . We may assume $\varphi$ is normalized (see the remark after 1.5).

We first claim $\mathscr{T}$ has an open set $1+c_{0} D_{\varphi}$ where $\varphi\left(c_{0} / b\right)<\infty$. First of all, $\mathscr{T}$ has an open set of the form $1+a D_{\varphi}$. Since $\mathscr{T}$ is 
a group topology, there exists $a^{\prime} \in F$ with $1+a^{\prime} D_{\varphi}$ open and $(1+$ $\left.a^{\prime} D_{\varphi}\right)^{-1} \subseteq 1+a D_{\varphi}$. But either $a$ or $a^{\prime}$ must have finite value; otherwise

$$
1-a=\left(1+a^{\prime}\left(a / a^{\prime}(1-a)\right)\right)^{-1} \in\left(1+a^{\prime} D_{\varphi}\right)^{-1} \sqsubseteq 1+a D_{\varphi},
$$

an impossibility. This takes care of the case $b=1$. Now suppose $b \neq 1$. Then by hypothesis we have an open set $1+c_{0} D_{\varphi}$ not containing $1+b$, i.e., with $\varphi\left(c_{0} / b\right) \leqq 1$. The claim is proved.

We are finished if $\varphi$ is nonarchimedean (since then $\varphi(n)=1$ so

$$
\left.1+b n^{-1} D_{\varphi} \supseteq 1+c_{0} D_{\varphi}\right) .
$$

Hence suppose $\varphi$ is Archimedean. Then $D_{\varphi}+D_{\varphi} \leqq 2 D_{\varphi}$. We can pick $s \in Z$ with $c_{0} D_{\varphi} \subseteq 2^{s} b D_{\varphi}$. There exists $c_{1} \in F$ with $1+c_{1} D_{\varphi}$ open and

$$
\left(1+c_{1} D_{\varphi}\right)^{2} \subseteq 1+c_{0} D_{\varphi}
$$

For each $d \in D_{\varphi}$,

$$
c_{1} d=2^{-2}\left(\left(1+c_{1} d\right)^{2}-\left(1-c_{1} d\right)^{2}\right) \in 2^{-1} c_{0} D_{\varphi} \leqq 2^{s-1} b D_{\varphi}
$$

so

$$
1+c_{1} D_{\varphi} \subseteq 1+2^{s-1} b D_{\varphi} .
$$

Repeating this process we find $c_{2} \in F^{\times}$with $1+c_{2} D_{\varphi}$ open and

$$
1+c_{2} D_{\varphi} \subseteq 1+2^{s-2} b D_{\varphi} .
$$

Continuing in this way we find $c \in F$ with $1+c D_{\varphi}$ open and $1+c D_{\varphi} \subseteq$ $1+b n^{-1} D_{\varphi}$.

REMARK 1.11. It can be shown that the set of $\varphi$-topologies on $F^{\times}$is naturally bijective with the set of proper ideals of the valuation ring

$$
A_{\varphi}=\{a \in F: \varphi(a) \leqq \varphi(n \cdot 1) \text { for some } n \in Z\} .
$$

(The bijection takes a $\varphi$-topology to $-1+\cap U$, where the intersection ranges over all neighborhoods $U$ of 1.)

The sets $1+n^{-1} D_{\varphi}(n \in Z, \varphi(n) \geqq 1)$ form a neighborhood base at 1 for a $\varphi$-topology which is coarser than all other $\varphi$-topologies (apply Lemma 1.10). Clearly, a subfield $E$ of $F$ is ultradense in $(F, \varphi)$ if and only if $E^{\times}$is dense in this coarsest $\varphi$-topology on $F^{\times}$. To prove Theorem 1.8 it therefore suffices to prove

LEMma 1.12. Let $(K, \psi)$ be an extension of $(F, \varphi) . \quad F^{\times}$is dense in the coarsest $\psi$-topology on $K^{\times}$if and only if $k_{\varphi}^{\times}$is dense in the coarsest $\bar{\psi}$-topology on $k_{\psi}^{\times}$and $\Gamma_{\varphi}=\Gamma_{\psi}$. 
Proof. $\quad \Leftrightarrow$ Let $a \in K^{\times}$and let $n$ be an integer, $\varphi(n \cdot 1) \geqq 1$. By hypothesis, there exists $b \in F$ with $0<\psi\left(a^{-1} b\right)<\infty$. Again, by hypothesis there exists $c \in F$ with

$$
a^{-1} b c+\psi^{-1}(0) \in 1+n^{-1} D_{\bar{\psi}}
$$

whence

$$
b c \in a\left(1+n^{-1} D_{\psi}\right) .
$$

This proves that in the coarsest $\psi$-topology every nonempty open set contains an element of $F$.

$\Leftrightarrow$ For each $a \in K^{\times}$there exists $b \in F^{\times}$with $b \in a\left(1+D_{\psi}\right)$, so $a / b \in \psi^{-1}\left(R^{\times}\right)$. This shows $\Gamma_{\varphi}=\Gamma_{\psi}$. Now let $a \in \psi^{-1}\left(R^{\times}\right)$and pick $n \in Z, \varphi(n \cdot 1) \geqq 1$. There exists $b \in F^{\times}$with $b \in a\left(1+n^{-1} D_{\psi}\right)$ so

$$
b+\varphi^{-1}(0) \in\left(a+\psi^{-1}(0)\right) \cdot\left(1+n^{-1} D_{\bar{\psi}}\right) .
$$

That is, $k_{\varphi}^{\times}$is dense in the coarsest $\bar{\psi}$-topology on $k_{\psi}^{\times}$.

REMARK 1.13. Suppose $\varphi$ is nonarchimedean. Then $D_{\varphi}$ is the maximal ideal of the valuation ring $A_{\varphi}=\{a \in F: \varphi(a) \leqq 1\} .(F, \varphi)$ is ultracomplete if and only if $A_{\varphi}$ is maximal [4, Example 2.2]. Also, $F$ is ultradense in an extension $(K, \psi)$ of $(F, \varphi)$ if and only if $A_{\psi}$ is an immediate extension of $A_{\varphi}$. (Proof. Assume $\varphi$ is normalized; now apply Theorem 1.8 and Remark 1.9.)

We prove half of Theorem 1.7 in

Proposition 1.14. $(F, \varphi)$ is ultracomplete if and only if $\varphi^{-1}(R)$ is maximal and $\left(k_{\varphi}, \bar{\varphi}\right)$ is ultracomplete.

Proof. If $\phi$ is nonarchimedean, then $A_{\varphi}$ is the localization of $\varphi^{-1}(R)$ at the prime ideal $D_{\varphi}$. The proposition then follows from Remark 1.13 and [16, pp. 113-114]. Now suppose $\varphi$ is Archimedean. Assume $(F, \varphi)$ is ultracomplete. The completeness of $F^{\times}$at the coarsest $\varphi$-topology implies the ultracompleteness of $\left(k_{\varphi}, \bar{\varphi}\right)$ (cf. Remark 1.9). Let $B \subseteq F$, say with $1 \in B$. Give $F^{\times}$the topology with neighborhood base at 1

$$
\left\{1+b \varphi^{-1}(0): b \in B\right\} \text {. }
$$

We must show $F^{\times}$is complete [4, Example 2.2]. If the set (2) has a smallest element (with respect to inclusion), then $F^{\times}$is clearly complete. Otherwise, for each $b \in B$ there exists $b^{\prime} \in B$ with

$$
b \varphi^{-1}(0) \supseteqq b^{\prime} D_{\varphi} \supseteqq b^{\prime} \varphi^{-1}(0) \text {. }
$$


Thus the topology on $F^{\times}$is a $\varphi$-topology (the sets $1+b D_{\varphi}, b \in B$, form a neighborhood base at 1 ). But $F^{\times}$is by hypothesis complete at every $\varphi$-topology.

Let us now assume, conversely, that $\varphi^{-1}(R)$ is maximal and $\left(k_{\varphi}, \bar{\varphi}\right)$ ultracomplete. Let $\mathscr{T}$ be a $\varphi$-topology on $F^{\times}$, say with $\left\{1+b D_{\varphi}: b \in B\right\}$ a neighborhood base at 1 . If $\left\{1+b \varphi^{-1}(0): b \in B\right\}$ has no minimum, then it is also a neighborhood base at 1 so by hypothesis and [4, Example 2.2], $F^{\times}$is complete at $\mathscr{T}$. Suppose, on the other hand, that it has a minimum. Then $\mathscr{T}$ admits a neighborhood base at 1 of the form $\left\{1+b n^{-1} D_{\varphi}: 0<n \in Z\right\}$ where $b \in B$ (use Lemma 1.10). Let $\left(d_{\lambda}\right)_{\lambda \in A}$ be a Cauchy net in $F^{\times}$(with respect to $\mathscr{T}$ ); we show it has a limit. Note $1+2^{-1} D_{\varphi}$ is in $\mathscr{T}$ (Lemma 1.10). Hence there exists $\mu \in \Lambda$ such that $\lambda>\mu$ implies

$$
d_{\lambda} d_{\mu}^{-1} \in\left(1+b D_{\varphi}\right) \cap\left(1+2^{-1} D_{\varphi}\right) .
$$

The net

$$
\left(b^{-1}\left(d_{\lambda} d_{\mu}^{-1}-1\right)+\varphi^{-1}(0)\right)_{\lambda \geqq \mu}
$$

is Cauchy in the $\bar{\varphi}$-topology on the additive group $k_{\varphi}$. (Subproof. For all $\lambda, \lambda^{\prime}>\mu, \varphi\left(d_{\lambda} d_{\mu}^{-1}\right)<2$ and

$$
\left.b^{-1}\left(d_{\lambda} \cdot d_{\mu}^{-1}-1\right)-b^{-1}\left(d_{\lambda} d_{\mu}^{-1}-1\right)=b^{-1}\left(d_{\lambda^{\prime}} d_{\lambda}^{-1}-1\right)\left(d_{\lambda} d_{\mu}^{-1}\right) .\right)
$$

The limit of the Cauchy net (3) can be put in the form

$$
b^{-1}\left(d d_{\mu}^{-1}-1\right)+\varphi^{-1}(0)
$$

where $d \in F^{\times}$. But then $d$ is the limit of $\left(d_{\lambda}\right)_{\lambda \in \Lambda}$. (Subproof. For $\lambda>\mu, \varphi\left(d_{\lambda} d_{\mu}^{-1}\right)>2^{-1}$. Now substitute $d$ for $d_{\lambda^{\prime}}$ in the identity (4).)

REMARK 1.15. Let $(k, \delta)$ be an ultracompletion of $\left(k_{\varphi}, \bar{\varphi}\right)$ (see Remark 1.9 for the existence of $(k, \delta))$. The valuation ring $\varphi^{-1}(R)$ extends to a maximal valuation ring $B$ with the same value group $\Gamma_{\varphi}$ and with residue class field ( $k_{\varphi}$-isomorphic to) $k$ [16, Chapter F, Theorem 3 (MacLane)]. Let $K$ be the field of fractions of $B$ and let $\psi$ be the extended absolute value obtained by composing the canonical place from $K$ to $k$ with $\delta$. Then $(K, \psi)$ is an ultracompletion of $(F, \varphi)$ (use Proposition 1.14 and Theorem 1.8).

Thus, $(F, \varphi)$ always has an ultracompletion. In general, ultracompletions are not unique [12, p. 381]. The family of ultracompletions of $(F, \varphi)$ carries more information about $F$ than a particular ultracompletion (see Remark 3.5).

Suppose $\varphi$ is Archimedean. Then between any two ultracompletions of $(F, \varphi)$ there is an $F$-isomorphism that preserves the extended absolute value (argue as in the proof of [8, Theorem 7]). We 
denote "the" ultracompletion by $\left(F_{\varphi}, \varphi\right) .\left(F_{\varphi}, \varphi\right)$ is isomorphic to a generalized power series field with residue class field $R$ or $C$ [8, Theorem 6] (cf. Example 1.1D).

We now complete the proof of Theorem 1.7. First suppose $F$ is ultradense in no proper extension of $(F, \varphi)$. Then the extension $(K, \psi)$ of Remark 1.15 above is not proper. Thus $(F, \varphi)$ is ultracomplete. Conversely, suppose $(F, \varphi)$ is ultracomplete. Suppose $F$ is ultradense in an extension $(E, \rho)$ of $(F, \varphi)$. First, $k_{\varphi}=k_{\rho}$ (use Proposition 1.14 and Theorem 1.8 to apply Remark 1.9). Also $\Gamma_{\varphi}=\Gamma_{\rho}$ (Theorem 1.8). Since $\varphi^{-1}(R)$ is maximal (Proposition 1.14), we have $F=E$. This completes the proof of Theorem 1.7.

The next two lemmas will be used in $\S 3$.

LemMA 1.16. Suppose $\pi: F \rightarrow E \cup\{\infty\}$ is a place and $\psi$ is an extended absolute value on $E$. Then $(F, \psi \circ \pi)$ is ultracomplete if and only if $(E, \psi)$ is ultracomplete and the valuation ring $\pi^{-1}(E)$ is maximal.

The composition $\psi \circ \pi$ should be understood to map $\pi^{-1}(\infty)$ to $\infty$. This lemma is an immediate corollary of [16, Chapter D, Propositions 7 and 8] and Theorem 1.7, which are special cases of it. It could also be proved through a slight modification of the proof of Proposition 1.14 .

Lemma 1.17. Situation as in Lemma 1.16. Let $L$ be a subfield of $F$. $L$ is ultradense in $(F, \psi \circ \pi)$ if and only if $L^{\times} \cdot \pi^{-1}\left(E^{\times}\right)=F^{\times}$ and $\pi(L) \cap E$ is ultradense in $(E, \psi)$.

This lemma follows from, and slightly generalizes, Theorem 1.8.

2. Harrison primes. Warner and Harrison define an "ultracompletion of a field at a finite or infinite prime" to be a "maximal immediate extension" of the primed field [7]. This approach is suggested by Krull's definition of a maximal field. We show here that the ultracompletions of a field at a Harrison prime are essentially just the ultracompletions of the field at the extended absolute values of the field associated with the prime, in the sense of the following definition.

DEFINITION 2.1. Let $\varphi$ be an extended absolute value and $P$ be a Harrison prime of $F$. We say $\varphi$ and $P$ are associated if and only if for all $a \in P$,

$$
\varphi(a+1)=\varphi(a)+1 .
$$

The reader should verify that the usual absolute value on the 
complex numbers $C$ is associated in the above sense with the prime $R^{2}$ consisting of all nonnegative real numbers. Also, the unique prime of a finite field (namely $\{0\}$ ) is associated with the unique absolute value (the value of every nonzero element is 1 ). One more example. An ordering of a field (which is a fortiori a Harrison prime) is associated with the extended absolute value that it canonically gives rise to (cf. Example (1.1D)).

Proposition 2.2. Let $\varphi$ and $P$ be as in 2.1. Then $\varphi$ and $P$ are associated if and only if one of the following two conditions occurs:

A. $\phi$ is the extended absolute value corresponding to a valuation ring with maximal ideal $P$ (cf. Example 1.1B);

B. $\varphi$ is the extended absolute corresponding to a complex place $\pi: F \rightarrow C \cup\{\infty\}$ with $\pi^{-1}\left(R^{2}\right)=$ Arch $P$ (cf. Example 1.1C).

Here Arch $P$ denotes the set of all $a \in F$ such that for some integer $n$ and all integers $m \geqq-1$ we have $n \cdot 1+m a \in P$. Harrison and Warner show Arch $P$ is a prime [7, Theorem 1.1].

A corollary of the above proposition is that each Harrison prime of $F$ has associated with it an extended absolute value and any such extended absolute value is a prime value. (To prove this use [7, Theorem 1.1] when $P$ is infinite and [6, Proposition 2.5] when $P$ is finite. This prime value is unique unless $P$ is complex.) In Remark 2.4 below, there is a computation of the set of Harrison primes associated with a given prime value; this set is not in general a singleton or even nonempty.

We now prove 2.2. First note that an infinite Harrison prime $P$ is associated with the prime value arising from a complex place $\pi$ if and only if $\pi(P) \geqq 0$ (i.e., $\pi(a) \in R^{2} \cup\{\infty\}$ for all $a \in P$ ). The sufficiency of conditions A (which implies $\varphi(P)=0$ ) and B (which implies $\pi(P) \geqq 0$ [7, Theorem 1.1]) is easily checked. Now suppose $P$ and $\varphi$ are associated.

Case 1. $P$ is finite, i.e., $P$ is the maximal ideal of a minimal valuation ring $[6$, p. 18]. Then $\varphi$ is nonarchimedean (for any positive integer $n$ with $n \cdot 1 \in P$ we have $\varphi(-n \cdot 1+1)=\varphi(n \cdot 1)+1)$. Indeed, $\varphi(P) \subseteq\{0, \infty\}$ (since $\varphi(b+1) \leqq \max \{\varphi(b), 1\}$ for all $b \in P$ ). By elementary valuation theory, if $P$ contains $\varphi^{-1}(0)$ then it is itself contained in $\varphi^{-1}(R)$ and so

$$
P \subseteq \varphi^{-1}(R) \cap \varphi^{-1}(0, \infty)=\varphi^{-1}(0) .
$$

But $P$ and $\varphi^{-1}(0)$ are not incomparable since otherwise there exists [3, Lemma 6.1] $b \in P$ with $\varphi(1+b)=0$, whence

$$
0=\varphi(1+b)=1+\varphi(b) \geqq 1 \text {. }
$$


Hence

$$
P \subseteq \varphi^{-1}(0) \subseteq D_{\varphi}
$$

But none of these containments can be proper ( $P$ is a maximal preprime). It follows that $\varphi^{-1}(R)$ is a valuation ring with associated extended absolute value $\varphi$ and maximal ideal $P$.

Case 2. $P$ is infinite (i.e., $1 \in P$ ). Then $\varphi$ is Archimedean and normalized (apply Definition 2.1 to obtain $\varphi(2)=2$ ). Hence $\varphi$ arises canonically from a complex place $\pi$. It suffices to show $\pi^{-1}(R) \supseteqq \operatorname{Arch} P$ since Arch $P$ is a prime. Recall that $J_{P}=\{a \in F: 1+Z a \subseteq P\}$ is the maximal ideal of a valuation ring of $F$, call it $A$, and $\operatorname{Arch} P=(A \cap P) \cup$ $J_{P}$. From Definition 2.1 and the elementary geometry of complex numbers we deduce that $\varphi\left(J_{P}\right)=\{0, \infty\}$ and $\pi(P) \geqq 0$. The former fact implies that the units of the valuation ring $\varphi^{-1}(R)$ are contained in the units of $A\left(a \in F\right.$ is not a unit in $A$ if and only if $a$ or $a^{-1}$ is in $J_{P}$, i.e., $a$ has value 0 or $\infty$ ). Thus by elementary valuation theory, $\varphi^{-1}(0) \supseteqq J_{P}$. Suppose $a \in \operatorname{Arch} P \backslash \varphi^{-1}(R)$. Then $a \in \operatorname{Arch} P \backslash J_{P}$ so for some positive integer $n$ we have $a^{-1}-n^{-1}$ in

$$
\left(\operatorname{Arch} P \backslash J_{P}\right) \cap \pi^{-1}\left(-n^{-1}\right) \text {. }
$$

But this set must be empty since $\operatorname{Arch} P \backslash J_{P} \subseteq P$ and $\pi(P) \geqq 0$. This shows Arch $P \subseteq \varphi^{-1}(R)$. Hence

$$
\operatorname{Arch} P=(A \cap P) \cup J_{P} \subseteq \varphi^{-1}(0) \cup\left(P \cap \varphi^{-1}(R)\right) \subseteq \pi^{-1}\left(R^{2}\right) .
$$

The proposition is proved.

THEOREM 2.3. Let $P$ be a Harrison prime of $F$. Let $(K, T)$ be a primed field extending $(F, P)$. Let $\psi$ be a prime value associated with T. Then $(K, T)$ is an ultracompletion of $(F, P)$ if and only if $(K, \psi)$ is an ultracompletion of $(F, \psi \mid F)$.

The second sentence of the above theorem means that $K / F$ is a field extension and $T$ is a prime of $K$ containing $P$.

It suffices to show that $(K, T)$ is ultracomplete (in the sense of [7]) if and only if $(K, \psi)$ is ultracomplete, and that the extension $(K, T)$ of $(F, P)$ is immediate if and only if $F$ is ultradense in $(K, \psi)$. Proposition 2.2 and Remark 1.9 provide the machinery for deducing the first of these facts from Theorem 1.7 and [7, Theorem 3.1], and the second from Theorem 1.8 and [7, $\S 3$, first paragraph].

REMARK 2.4. If $\varphi$ is a nonarchimedean prime value, then $D_{\varphi}$ is the unique Harrison prime associated with $\varphi$. Now suppose $\varphi$ is an 
Archimedean prime value, say canonically arising from the complex place $\pi$. No primes are associated with $\varphi$ unless $\pi(F) \cap R^{2}$ is a prime of $\pi(F) \cap C$ [7, Theorem 1.1], so suppose this is the case. Note that if $P$ is associated with $\varphi$ then $P \cap(-P)$ is a prime ideal of $\varphi^{-1}(R)$. Now let $\zeta$ be any prime ideal of $\varphi^{-1}(R)$. Let $A$ denote the localization of $\varphi^{-1}(R)$ at $\zeta$. Then the set of primes $P$ of $F$ associated with $\varphi$ and with $P \cap(-P)=\zeta$ is bijective (by the map $P \rightarrow P \backslash \zeta$ ) with the set of subgroups $G$ of the group of units of $A$ maximal with respect to having

$$
G \cap \pi^{-1}\left(C^{\times}\right)=\pi^{-1}\left(R^{\times 2}\right) .
$$

This result sets a theorem of Harrison and Warner into the language of extended absolute values. (Sketch of proof. The maximality condition on the group $G$ above guarantees that the image of $G$ in $\Gamma_{\varphi}$ generates the isolated subgroup associated with $\zeta[16$, Chapter C, Theorem 1]. The remark now follows from [8, Theorem 2.2] by Noether's isomorphism theorems and elementary valuation theory [16, Chapter C].)

Suppose the prime $P$ of $F$ is associated with the prime value $\varphi$. Let $(K, \psi)$ be an ultracompletion of $(F, \varphi)$. There exists a prime $T$ of $K$ associated with $\psi$ and containing $P$ (take $T=D_{\psi}$ if $\psi$ is nonarchimedean and apply the above result otherwise). That is, $(K, T)$ is an ultracompletion of $(F, P)$ in the sense of [7].

3. Quadratic forms. Throughout this section we assume $F$ does not have characteristic two. Two quadratic forms over $F$ are said to be equivalent as quadratic forms over $F$ when they have the same number of variables and each can be obtained from the other by an $(F-)$ linear change of variables. (See [17] for equivalent concepts and definitions.)

Let $Q(x)$ be a simple transcendental extension of the rational numbers $Q$.

THEOREM 3.1. Two quadratic forms over $Q(x)$ are equivalent as quadratic forms over $Q(x)$ if and only if they are equivalent as quadratic forms over $K$ for every ultracompletion $(K, \psi)$ of $Q(x)$ at a prime value of $Q(x)$.

In the above theorem we understand $K$ to range over all ultracompletions at all prime values. Recall that the prime values of $F$ are exactly the normalized members of the extended prime spots of $F[3, \S 5]$.

Let us temporarily call $F$ special if the above theorem is valid with $Q(x)$ replaced by $F$. Not all fields are special (see the examples 
below). Pythagorean fields are special (see [17, Lemma 2.2.4] and Theorem 3.3). The Hasse-Minkowski theorem says that global fields not of characteristic two are special [17].

Let $W(F)$ denote the Witt ring of equivalence classes of anisotropic quadratic forms over $F$ [17, Proposition 1.6.5]. Each field extension $K / F$ induces a ring homomorphism $W(F) \rightarrow W(K)$. For $f \in W(F)$, let $f \otimes K$ denote the image of $f$ in $W(K)$. Let

$$
W(F) \longrightarrow \Pi_{K} W(K)
$$

be the product of all such maps as $K$ ranges over all ultracompletions of $F$ at all prime values of $F$. Now, two quadratic forms over $F$ with the same number of variables are equivalent if and only if their radicals have the same dimension and the difference (in $W(F)$ ) of their anisotropic parts is zero [17, Corollaries 1.3.10 and 1.5.5]. Hence $F$ is special if and only if the map (5) above is injective.

The Hasse-Minkowski theorem and the next proposition together imply that every simple extension of a global field, and hence in particular $Q(x)$, is special.

Proposition 3.2. $F(x)$ is special if every simple algebraic extension of $F$ is special.

Proof. Let $0 \neq f \in W(F(x))$. We must show $0 \neq f \otimes K$ for some ultracompletion $(K, \psi)$ of $F(x)$ with $\psi$ a prime value. First suppose there exists $h \in W(F)$ with $h \otimes F(x)=f$. Since $F$ is special, there exists an ultracompletion $(K, \psi)$ of $F$ with $\psi$ a prime value and with $h \otimes K \neq 0$. Let $\delta$ be the prime value obtained by composing $\psi$ with the canonical place from the field of formal series $K((x))$ to $K$. Then $(K((x)), \delta)$ is an ultracompletion of $F(x)$ by Lemmas 1.16 and 1.17 . Also $f \otimes K((x)) \neq 0$, since the canonical map $W(K) \rightarrow W(K((x)))$ is injective [14, Theorem of Springer, p. 334].

Now suppose there does not exist $h \in W(F)$ with $f=h \otimes F(x)$. Then there exists a monic irreducible polynomial $\pi \in F[x]$ such that

$$
0 \neq \sum_{i}\left\langle u_{i}+(\pi)\right\rangle \in W(F[x] /(\pi))
$$

where

$$
\sum_{i} \pi u_{i} x_{i}^{2}+\sum_{i} w_{i} y_{i}^{2}
$$

is an anisotropic form representing $f$ with each $u_{i}$ and $w_{i}$ a polynomial in $F[x]$ not divisible by $\pi$ [14, Theorem 5.3]. (The $x_{i}$ and $y_{i}$ above are independent indeterminants. For $\alpha \in F[x] /(\pi),\langle\alpha\rangle$ denotes the image in $W\left(F[x] /(\pi)\right.$ ) of the quadratic form $\alpha y^{2}$.) By hypothesis $F[x] /(\pi)$ has an ultracompletion $\left(K_{0}, \psi_{0}\right)$ where $\psi_{0}$ is a prime value and 


$$
\sum_{i}\left\langle u_{i}+(\pi)\right\rangle \otimes K_{0} \neq 0
$$

Give $F(x)$ the $\pi$-adic valuation; it then admits a maximal extension $K$ with residue class field $F$-isomorphic to $K_{0}$ and with the same value group as $F(x)$. Let $\psi$ be the composition of $\psi_{0}$ and the canonical place $K \rightarrow K_{0}$. Then $(K, \psi)$ is an ultracompletion of $F(x)$ (cf. Lemmas 1.16 and 1.17). $f \otimes K$ is nonzero since its canonical image in $W\left(K_{0}\right)$ is the element (6) above [14, Corollary 5.1]. This proves the proposition.

Let $W_{r e d}(F)$ denote the reduced Witt ring of $F$, i.e., the Witt ring modulo its nil radical. For any field extension $K$ of $F$ we have a natural map $W_{r e d}(F) \rightarrow W_{r e d}(K)$. Recall that for each Archimedean prime value $\varphi$ on $F$, we denote by $F_{\varphi}$ the (unique up to $F$-isomorphism) ultracompletion of $(F, \varphi)$ (cf. 1.15).

THEOREM 3.3. The natural map

$$
W_{\text {red }}(F) \longrightarrow \Pi_{\varphi} W_{\text {red }}\left(F_{\varphi}\right)
$$

(product over all Archimedean prime values $\varphi$ ) is injective.

Proof. If $F$ is not formally real, the nil radical of $W(F)$ consists of the forms of even dimension-index [17, Corollary 2.3.3]. Our result follows immediately in this case.

Now suppose $F$ is formally real and $f \in W(F)$ is not nilpotent. Then there exists an ordering $P$ of $F$ with respect to which $f$ has nonzero signature [15, Theorem 2.2]. Let $\pi$ and $\phi$ denote the real place and prime value associated with this ordering (cf. Example 1.1D). Let $\pi^{\prime}$ denote the canonical extension of $\pi$ to $F_{\varphi}$. $P$ has a unique extension to $F_{\varphi}\left(a \in F_{\varphi}^{\times}\right.$is positive if and only if $\pi^{\prime}(a / b)$ is positive for every $b \in P$ with $\pi^{\prime}(a / b)$ finite and nonzero. The existence of such $b$ follows from Theorem 1.8.) Since $f \otimes F_{\varphi}$ has the same (nonzero!) signature as $f$, it must be nonnilpotent. The theorem is proved.

REMARK 3.4. Theorem 3.3 is little more than a setting of Pfister's [15, Theorem 2.2] into the language of extended absolute values. Pfister's theorem says that if $F$ is formally real, then the natural map

$$
W_{r e d}(F) \longrightarrow \Pi_{P} W\left(F_{P}\right)
$$

is injective (the product is over all orderings $P$ of $F . \quad F_{P}$ denotes the real closure of $F$ at the ordering $P$. Note that $W\left(F_{P}\right) \cong Z$, the isomorphism assigning to each element of $W\left(F_{P}\right)$ its signature.) Our hope is that the image of the map (7) will be easier to compute than the image of the map (8). Either computation would give a fairly detailed picture of $W_{r e d}(F)$. (Note that the structure of $W\left(F_{\varphi}\right)$ 
is known for real $\varphi$; see formula (9) below.) The computation of the image of $(8)$ is discussed in $[3, \S 4 ; 11]$.

We now indicate briefly how to recover Pfister's result from Theorem 3.3. Let $\varphi$ be any real prime value on $F$, i.e., one arising from a place into $R$. Each ordering of $F$ associated with $\varphi$ has a unique extension to $F_{\varphi}$ and all orderings of $F_{\varphi}$ are obtained in this way. (The "unique extension" was described in the proof of 3.3. The second assertion follows from the last paragraph of $\S 4$.) That is, any $f \in W(F)$ has zero signature at all orderings associated with $\varphi$ if and only if $f \otimes F_{\varphi}$ has zero signature at all orderings of $F_{\varphi}$. Hence (and here we use Theorem 3.3), it is enough to show the map (8) is injective whenever $F$ is ultracomplete at a real prime value. So now suppose $F=F_{\varphi}$. Fix an ordering $P$ associated with $\varphi$. For each $a \in F^{\times}$, let $\underline{a}$ denote the canonical image of $a$ in $\Gamma_{\varphi} / \Gamma_{\varphi}^{2}$ and let $\sigma_{P}(a)$ denote 1 or -1 according as $a \in P$ or $a \notin P$. By mapping $a x^{2}$ to the element $\sigma_{P}(\alpha) \cdot \underline{\alpha}$ of the integral group ring of $\Gamma_{\varphi} / \Gamma_{\varphi}^{2}$ we induce an isomorphism

$$
W(F) \longrightarrow Z\left[\Gamma_{\varphi} / \Gamma_{\varphi}^{2}\right]
$$

If we map $\Gamma_{\varphi} / \Gamma_{\varphi}^{2}$ to its second dual, we induce [12, p. 104] a ring injection [1, Theorem 1.2]

$$
Z\left[\Gamma_{\varphi} / \Gamma_{\varphi}^{2}\right] \longrightarrow \operatorname{Map}\left(\operatorname{Hom}\left(\Gamma_{\varphi} / \Gamma_{\varphi}^{2}, \pm 1\right), Z\right) \text {. }
$$

Finally, the isomorphism of [2, Proposition] induces an isomorphism

$$
\gamma: \operatorname{Map}\left(\operatorname{Hom}\left(\Gamma_{\varphi} / \Gamma_{\varphi}^{2}, \pm 1\right), Z\right) \longrightarrow \Pi_{T} W\left(F_{T}\right)
$$

(product over all orderings $T$ of $F$. If we identify $W\left(F_{T}\right)$ with $Z$ and $\{ \pm 1\}$ with $Z_{2}$, then $\gamma$ is given by $\gamma(f)(T)=f(\langle T, P\rangle)$.) One can now check that the map (8) is the composition of the injections (9), (10), and (11). (Use here that $\langle P, T\rangle(\underline{a})=\sigma_{P}(a) \sigma_{T}(a)$.)

We now set into the language of prime values a result of Harrison and Warner [7, Theorem 3.2]. It says, roughly, that the existence of an "approximate" (as measured by $\varphi$ ) solution to a quadratic equation over $F$ is equivalent to the existence of exact solutions in the family of ultracompletions of $(F, \varphi)$. This result generalizes and strengthens the local squares theorem.

REMARK 3.5. Let $\varphi$ be a prime value on $F$. There exists a $\varphi$-topology on $F^{\times}$with respect to which the closure of $F^{\times 2}$ is precisely the set of elements of $F^{\times}$which are squares in every ultracompletion of $(F, \varphi)$. This topology may be taken to be the coarsest $\varphi$-topology separating 1 from 5 , unless $k_{\varphi}$ has characteristic 2 and admits no quadratic extensions. In this latter case we may take the topology 
to be the coarsest $\varphi$-topology separating 1 from $1+b$ for every $b \in D_{\varphi} \mid 4 D_{\varphi}$ whose image in $\Gamma_{\varphi}$ is odd (i.e., does not have a square root in $\Gamma_{\varphi}$, writing $\Gamma_{\varphi}$ multiplicatively).

Proof (sketch). Suppose $k_{\varphi}$ has characteristic 2 and admits no quadratic extensions. Give $F^{\times}$the $\varphi$-topology indicated above. Let $(K, \psi)$ be an ultracompletion of $(F, \varphi)$. Let $w$ be the unique (additively written) valuation on $K$ with $w\left(D_{\psi}\right)>0$. First suppose $a \in F^{\times}$ is in the closure of $F^{\times 2}$. Since $F^{\times 2}$ meets $a\left(1+D_{\varphi}\right), w^{s q}\left(a K^{\times 2}\right) \geqq 0$ (we use the notation of [4] or [7, §3]). $w(4)$ and 0 cannot equal $w^{s q}\left(a K^{\times 2}\right)$ [4, Theorem 1.1]. Also, for any $b \in D_{\varphi} \backslash 4 D_{\varphi}$ with $w(b)$ odd, we have $w^{s q}\left(a K^{\times 2}\right)>w(b)$ since by hypothesis $F^{\times 2}$ meets $a\left(1+b D_{\varphi}\right)$. Hence $w^{s q}\left(a K^{\times 2}\right)=\infty$, i.e., $a \in K^{\times 2}$. Now suppose conversely that $a \in F^{\times}$ is a square in every ultracompletion of $(F, \varphi)$. Clearly then, $w(a)$ has even value in $\Gamma_{\varphi}$ and hence (since $k_{\varphi}$ is perfect) $a\left(1+D_{\varphi}\right)$ meets $F^{\times 2}$. Now suppose $b \in D_{\varphi} \backslash 4 D_{\varphi}$ has $w(b)$ odd (in $\Gamma_{\varphi}$ ). By [7, Theorem 3.2], $a F^{\times 2}$ meets $1+b D_{\varphi}$ so $a\left(1+b D_{\varphi}\right)$ meets $F^{\times 2}$. That is, $a$ is in the closure of $F^{\times 2}$.

The other cases are similar, but easier. (For Archimedean $\varphi$, use the proof of [7, Theorem 3.2].) The remark above generalizes (same proof) to any extended absolute value $\varphi$ such that if $1+1 \in D_{\varphi}$ (so $\varphi$ is nonarchimedean), then the residue class field of $\{a \in F: \varphi(a) \leqq 1\}$ is perfect.

We now give two examples of fields which are not special. $F$ is special only if every element in $F$ which is a square in every ultracompletion of $F$ is itself a square in $F$ (since for each $a \in F$, the quadratic forms $a x^{2}$ and $x^{2}$ are equivalent if and only if $a \in F^{\times 2}$ ). Our first example is of an algebraic extension of $Q$ not admitting such a "global squares theorem".

EXAMPle 3.6. Let $F=Q[\{\sqrt{(2 p)}: p$ is a prime number $\}]$. Then $2 \notin F^{\times 2}$. For otherwise there exist distinct odd primes $p_{1}, \cdots, p_{n}$ with $\sqrt{2}$ in the field $Q\left[\sqrt{\left(2 p_{1}\right)}, \cdots, \sqrt{\left(2 p_{n}\right)}\right]$. But then this field equals $Q\left[\sqrt{2,} \sqrt{p_{1}}, \cdots, \sqrt{p_{n}}\right]$, which is impossible since these two fields clearly have different dimensions as vector spaces over $Q$. (I gratefully acknowledge the help of the several people who made this clear to me.) On the other hand, 2 is clearly a square in every ultracompletion of $F$ at an Archimedean prime value. Further, suppose $\phi$ is a nonarchimedean prime value, say with $k_{\varphi}$ of characteristic $p$. By the Dirichlet theorem on arithmetic progressions there exists a prime number $q$ of the form $1+4 p n$ with $n \in Z$. By the local squares theorem $2=(2 q) / q$ is a square in every ultracompletion of $(F, \varphi)$.

This example is essentially unchanged if one replaces "ultracom- 
pletions at prime values" by, for example, "topological completions at nontrivial absolute values" or "Henselizations at prime values" (cf. §4).

We thank D. K. Harrison for the idea for our next example of a finitely generated field which is not special. It is based on the observation that if $F$ is special and not formally real, then $W(F)$ has characteristic at most 8 . For, $W(F)$ is then by definition a subdirect product of rings each of which has characteristic at most 8. (Every field ultracomplete at a nonreal prime value contains a copy of either $C$ or some $p$-adic field.)

EXAMPLE 3.7. Let

$$
F=Q\left(x_{1}, x_{2}, \cdots, x_{8}\right)\left[\sqrt{\left(-x_{1}^{2}-x_{2}^{2}-\cdots-x_{8}^{2}\right)}\right]
$$

where the $x_{i}$ are independent indeterminants. Then $F$ is a finitely generated nonreal field with $W(F)$ of characteristic 16 [17, Theorem 2.5.4] and hence, by the above remarks, is not special.

J. Schneider constructed the first example of a nonspecial field that I know of (unpublished).

4. Henselizations. We sketch without proofs some results on "Henselizations" of fields at extended absolute values. Let $\phi$ be an extended absolute value on $F$.

It is easy to show that if $K$ is a field extension of $F$, then $\varphi$ has an extension to an extended absolute value on $K$. We call $\varphi$ Henselian when it has exactly one extension to $F^{s e p}$, the separable closure of $F$. Then $\varphi$ is Henselian if and only if $A_{\varphi}$ is a Henselian valuation ring and either $\varphi$ is nonarchimedean (cf. 1.9) or $k_{\varphi}[\sqrt{(-1)}]$ is algebraically closed. Consequently, ultracomplete fields are Henselian. Also, suppose $\varphi$ is a real prime value. Then $F$ is real closed if and only if $\varphi$ is Henselian and $\Gamma_{\varphi}$ is divisible.

Many basic facts about Henselian extended absolute values are easy consequences of the corresponding facts about Henselian valuations. E.g., from [16, Chapter F, Corollary 2 (Ostrowski)] we have: If $\phi$ is Henselian, then $F$ is separably closed in the topological completion of $F$ at the topology induced by $\varphi$ (cf. $[3, \S 6])$. Krasner's lemma [16, Chapter F, Corollary 1] also generalizes easily.

By a Henselization of $(F, \varphi)$ we mean a minimal Henselian extension of $(F, \varphi)$. Let $\varphi^{\prime}$ be any extension of $\varphi$ to $F^{s e p}$. Let $K$ denote the subfield of $F^{\text {sep }}$ of elements fixed under the group of $F$-automorphisms of $F^{s e p}$ preserving $\phi^{\prime}$ (i.e., with $\phi^{\prime} \circ \sigma=\phi^{\prime}$ ). Then $\left(K, \varphi^{\prime} \mid K\right)$ is a Henselization of $(F, \varphi)$. In fact it is the "only one". Uniqueness follows from the strong universal property (compare with [16, p. 175]): Let $L$ be a (possibly transcendental) extension of $F$. 
Let $\rho$ be a Henselian extension of $\varphi$ to $L$. Then there is a unique $F$-isomorphism $\sigma$ of $K$ into $L$ with $\rho \sigma=\phi^{\prime} \mid K$.

Now suppose $\varphi$ and $\psi$ are incomparable normalized extended absolute values on $F$, with $\varphi$ Henselian. Then the Henselization of $\left(k_{\psi}, \bar{\psi}\right)$ is algebraically closed. (Corollary: A field with a real Henselian prime value admits exactly one place into $R$.) The isolated subgroup of $\Gamma_{\psi}$ associated with the valuation ring $A_{\varphi} \cdot A_{\psi}$ is divisible. (See [9] and [16, Chapter H, Corollary 1 (Schmidt)] for related results.)

\section{REFERENCES}

1. E. Artin, Galois Theory, Notre Dame Mathematical Lectures, No. 2, Notre Dame, Indiana, 1959.

2. Ron Brown, Real places and ordered fields, Rocky Mountain J. Math., 1 (1971), 633-636.

3. - An approximation theorem for extended prime spots, Canad. J. Math., 24 (1972), 167-184.

4. Ron Brown and H. D. Warner, Quadratic extensions of linearly compact fields, Trans. Amer. Math. Soc., 163 (1972), 379-399.

5. I. Fleischer, Maximality and ultracompleteness in normed modules, Proc. Amer. Math. Soc., 9 (1968), 151-157.

6. D. K. Harrison, Finite and infinite primes for rings and fields, Mem. Amer. Math. Soc., 68 (1966).

7. D. K. Harrison and H. D. Warner, Infinite primes of fields and completions, Pacific J. Math., 45 (1973), 201-216.

8. I. Kaplansky, Maximal fields with valuations, Duke Math. J., 9 (1942), 303-321.

9. I. Kaplansky and O.F.G. Schilling, Some remarks on relatively complete fields, Bull. Amer. Math. Soc., 48 (1942), 744-747.

10. J. Kelley, General Topology, Van Nostrand, Princeton, New Jersey, 1955.

11. M. Knebusch, A. Rosenberg, and R. Ware, Siructure of Witt rings, quotients of abelian groups, and orderings of fields, Bull. Amer. Math. Soc., 77 (1971), 205-210.

12. S. MacLane, The uniqueness of the power series representation of fields with valuations, Annals of Math. Ser., (2), 39 (1938), 370-382.

13. - Homology, Academic Press, New York, 1963.

14. J. Milnor, Algebraic K-theory and quadratic forms, Invent. Math., 9 (1970), 318-344.

15. A. Pfister, Quadratische Formen in beliebigen Körpern, Invent. Math., 1 (1966), 116-132.

16. P. Ribenboim, Théorie des Valuations, Les Presses de l'Université de Montréal, Montreal, 1964.

17. W. Sharlau, Quadratic Forms, Queen's papers in pure and applied mathematics, No. 22, Queen's University, Kingston, Ontario, 1969.

Received February 16, 1973. This paper was supported by a postdoctoral fellowship at Simon Fraser University (partially supported by the Canadian NRC) and by NSF grant \# GP-33034.

Simon Fraser University, Burnaby, British Columbia

AND

UNIVERSITY OF HAWAII 



\section{PACIFIC JOURNAL OF MATHEMATICS}

\section{EDITORS}

RICHARD ARENS (Managing Editor)

University of California

Los Angeles, California 90024
J. DUGUNDJI*

Department of Mathematics

University of Southern California

Los Angeles, California 90007

D. Gilbarg and J. Milgram

Stanford University

Stanford, California 94305
University of Washington

Seattle, Washington 98105

ASSOCIATE EDITORS
E. F. BECKENBACH
B. H. NeumanN
F. WoLF
K. YosHIDA

\section{SUPPORTING INSTITUTIONS}

\author{
UNIVERSITY OF BRITISH COLUMBIA \\ CALIFORNIA INSTITUTE OF TECHNOLOGY \\ UNIVERSITY OF CALIFORNIA \\ MONTANA STATE UNIVERSITY \\ UNIVERSITY OF NEVADA \\ NEW MEXICO STATE UNIVERSITY \\ OREGON STATE UNIVERSITY \\ UNIVERSITY OF OREGON \\ OSAKA UNIVERSITY
}

\author{
UNIVERSITY OF SOUTHERN CALIFORNIA \\ STANFORD UNIVERSITY \\ UNIVERSITY OF TOKYO \\ UNIVERSITY OF UTAH \\ WASHINGTON STATE UNIVERSITY \\ UNIVERSITY OF WASHINGTON
* * * *
AMERICAN MATHEMATICAL SOCIETY \\ NAVAL WEAPONS CENTER
}

The Supporting Institutions listed above contribute to the cost of publication of this Journal, but they are not owners or publishers and have no responsibility for its content or policies.

Mathematical papers intended for publication in the Pacific Journal of Mathematics should be in typed form or offset-reproduced, (not dittoed), double spaced with large margins. Underline Greek letters in red, German in green, and script in blue. The first paragraph or two must be capable of being used separately as a synopsis of the entire paper. Items of the bibliography should not be cited there unless absolutely necessary, in which case they must be identified by author and Journal, rather than by item number. Manuscripts, in duplicate if possible, may be sent to any one of the four editors. Please classify according to the scheme of Math. Rev. Index to Vol. 39. All other communications to the editors should be addressed to the managing editor, or Elaine Barth, University of California, Los Angeles, California, 90024.

100 reprints are provided free for each article, only if page charges have been substantially paid. Additional copies may be obtained at cost in multiples of 50 .

The Pacific of Journal Mathematics is issued monthly as of January 1966. Regular subscription rate: $\$ 72.00$ a year (6 Vols., 12 issues). Special rate: $\$ 36.00$ a year to individual members of supporting institutions.

Subscriptions, orders for back numbers, and changes of address should be sent to Pacific Journal of Mathematics, 103 Highland Boulevard, Berkeley, California, 94708.

PUBLISHED BY PACIFIC JOURNAL OF MATHEMATICS, A NON-PROFIT CORPORATION

Printed at Kokusai Bunken Insatsusha (International Academic Printing Co., Ltd.), 270, 3-chome Totsuka-cho, Shinjuku-ku, Tokyo 160, Japan.

* C. R. DePrima California Institute of Technology, Pasadena, CA 91109, will replace J. Dugundji until August 1974.

Copyright (C) 1973 by Pacific Journal of Mathematics

Manufactured and first issued in Japan 


\section{Pacific Journal of Mathematics}

\section{Vol. 51, No. $2 \quad$ December, 1974}

Robert F. V. Anderson, Laplace transform methods in multivariate spectral theory .................................................. 339

William George Bade, Two properties of the Sorgenfrey plane . . . . . . . . . . . . 349

John Robert Baxter and Rafael Van Severen Chacon, Functionals on continuous

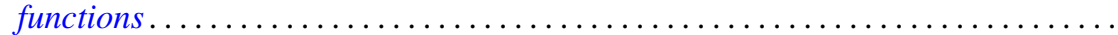

Phillip Wayne Bean, Helly and Radon-type theorems in interval convexity

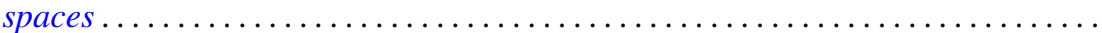

James Robert Boone, On k-quotient mappings $\ldots \ldots \ldots \ldots \ldots \ldots \ldots \ldots \ldots$

Ronald P. Brown, Extended prime spots and quadratic forms . . . . . . . . . . . .

William Hugh Cornish, Crawley's completion of a conditionally upper continuous lattice .............................................

Robert S. Cunningham, On finite left localizations ...................

Robert Jay Daverman, Approximating polyhedra in codimension one spheres

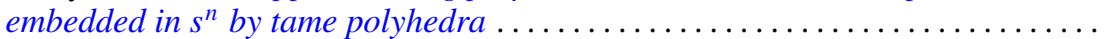

Burton I. Fein, Minimal splitting fields for group representations . . . . . . . . . . . .

Peter Fletcher and Robert Allen McCoy, Conditions under which a connected

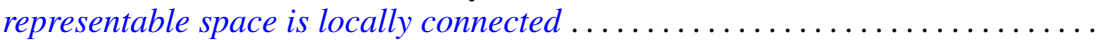

Jonathan Samuel Golan, Topologies on the torsion-theoretic spectrum of a noncommutative ring...

Manfred Gordon and Edward Martin Wilkinson, Determinants of Petrie matrices.

Alfred Peter Hallstrom, A counterexample to a conjecture on an integral condition for determining peak points (counterexample concerning peak points)........

E. R. Heal and Michael Windham, Finitely generated $F$-algebras with applications to Stein manifolds.

Denton Elwood Hewgill, On the eigenvalues of a second order elliptic operator in an unbounded domain ............................

Charles Royal Johnson, The Hadamard product of $A$ and $A^{*}$.

Darrell Conley Kent and Gary Douglas Richardson, Regular completions of Cauchy spaces.

Alan Greenwell Law and Ann L. McKerracher, Sharpened polynomial approximation

Bruce Stephen Lund, Subalgebras of finite codimension in the algebra of analytic functions on a Riemann surface. .

Robert Wilmer Miller, TTF classes and quasi-generators . .

Roberta Mura and Akbar H. Rhemtulla, Solvable groups in which every maximal partial order is isolated ....

Isaac Namioka, Separate continuity and joint continuity...

Alan Saleski, Entropy of self-homeomorphisms of statistical pseudo-metric

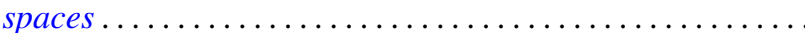

H. A. Seid, Cyclic multiplication operators on $L_{p}$-spaces .....

H. B. Skerry, On matrix maps of entire sequences ............

John Brendan Sullivan, A proof of the finite generation of invariants of a normal

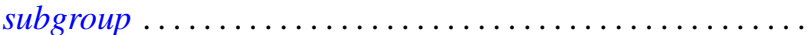

John Griggs Thompson, Nonsolvable finite groups all of whose local subgroups are

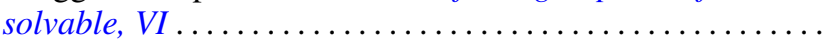

